\title{
Do Not Resuscitate orders and ethical decisions in a neonatal intensive care unit in a Muslim community
}

\author{
D E da Costa, H Ghazal, Saleh Al Khusaiby
}

Arch Dis Child Fetal Neonatal Ed 2002;86:F115-F1 19

\begin{abstract}
Aims: To evaluate the need for Do Not Resuscitate (DNR) orders in a tertiary referral centre for neonatal intensive care, the criteria used in making these decisions, and the applicability of the Muslim ethical stance among parents in an Islamic community.

Methods: A prospective evaluation of all DNR decisions in the neonatal intensive care unit at the Royal Hospital in Oman, over a one year period between November 1999 and October 2000. This included decision criteria, and parental responses and expectations.

Results: Of 659 admissions to the neonatal intensive care unit during this period, DNR orders were written in $39(6 \%)$ instances. Most related to congenital malformations (24/39,62\%). In those in whom ventilation was commenced $(19 / 39,49 \%)$ withdrawal was not culturally acceptable and expressly permitted in only $11 \%$. For those in whom ventilation was not commenced $(20 / 39,51 \%), 70 \%$ agreed not to put their child on the ventilator if they did require it. Presence of extended family support (grandparents) and clergy was extremely useful.

Conclusions: Asking parents alone to be explicitly involved or take full responsibility for decisions involving life and death is not culturally or socially acceptable in this community. Presence of extended family, and indirectly sounding out and taking into account their wishes, is more appropriate after assessing the resources and support services available.
\end{abstract}

See end of article for authors' affiliations

A lthough technology exists to sustain vital functions of almost any neonate, albeit for a short time, not every patient is a candidate for initiating and maintaining life support. All neonatal intensive care unit (NICU) physicians are called on to make "Do Not Resuscitate" (DNR) orders on a regular basis. Problems specific to the NICU relate to malformations of the fetus including lesions incompatible with life, and with problems relating to the limits of viability. Immense advances in neonatal intensive care over the past 25 years, especially in the developed nations, mean that more and more of these infants are surviving, with or without severe handicap. ${ }^{12}$ Community expectations have risen, sometimes inappropriately, and pressure may be put on health care providers to attempt salvage of nearly every live born baby. This raises many ethical and practical questions. Standards from the developed nations are often transposed onto other communities, where salvaging these infants may be unwarranted and community support services limited.

Religious and cultural issues often play a more vital role in decision making by parents and physicians than economic considerations, especially in Arab nations. Physicians are often reluctant to even broach the subject of DNR with parents, believing it will not be accepted, may cause a loss of trust in the physician, or "it is unfair to ask parents to be involved in the decision making process involving life and death". This has led to a significant increase in the number of handicapped survivors, and to a shortage of intensive care beds, as a result of inappropriate intensive care being given for an inordinately long time.

We assessed the frequency of the need for DNR orders in an NICU setting, the criteria used in vital support limitation decisions, parental expectations and response, and the outcome of these infants, in a Muslim Arab community. While there are numerous guidelines and personal viewpoints published from Britain and North America, ${ }^{3}$ no data have been published, to the best of our knowledge, from this population group, and very few data from neonatal units anywhere else on parental responses to end of life decisions.

\section{METHODS}

We prospectively assessed all patients who had the need for a DNR order considered in an Omani NICU between November 1999 and October 2000. The Royal Hospital is a 30 bedded level 3 NICU, catering for a high risk obstetric service of about 5000 deliveries annually; it serves as a referral unit for an additional 30000 deliveries from the country. Referrals of neonates from other units are mainly for complex cardiac and neonatal surgical problems, but occasionally for prematurity and its complications. More than $95 \%$ of patients, $70 \%$ of physicians, and $50 \%$ of nursing staff are Muslim.

Data were gathered primarily to answer the following questions:

(1) The frequency of DNR orders in a tertiary care NICU.

(2) The circumstances and criteria used to initially consider writing a DNR order.

(3) Parental expectations and response to limiting or withdrawing life support in the context of Islamic ethics.

(4) The eventual short term outcome of these patients (death or discharge home).

When a member of the care giving team felt a DNR order should be considered, a conference was held to determine the reason and obtain nursing, medical, and social workers' opinion and concurrence. Once this was obtained, counselling sessions with both parents or the father alone were done by at least two care givers, of whom one was the primary staff physician and the other the bedside nurse taking care of the infant. A native Arabic speaking physician was always present during counselling. Parental expectations were recorded, as was the type of care before and after writing a DNR order. Guidelines for managing the patient after a DNR order was

Abbreviations: DNR, Do Not Resuscitate; NICU, neonatal intensive care unit 
Table 1 Major diagnosis in DNR patients $(n=39)$

\begin{tabular}{|c|c|}
\hline Type of lesion & No. \\
\hline Central nervous system & 15 \\
\hline Meningomyelocele & 2 \\
\hline Grade 4 bilateral IVH & 5 \\
\hline Severe birth asphyxia & 2 \\
\hline Anencephaly & 1 \\
\hline Congenital hydrocephalus & 1 \\
\hline Neuronal migration defect & 2 \\
\hline Holoprosencephaly & 1 \\
\hline Cortical infarction & 1 \\
\hline Cardiovascular system & 9 \\
\hline Hypoplastic left heart & 3 \\
\hline Pulmonary atresia & 2 \\
\hline Complex cyanotic disease & 2 \\
\hline Interrupted aortic arch & 2 \\
\hline Respiratory system & 4 \\
\hline Pulmonary hypoplasia & 1 \\
\hline PPHN/MAS & 1 \\
\hline Severe chronic lung disease with respiratory failure & 2 \\
\hline Renal system & 2 \\
\hline Prune belly syndrome/renal failure & 1 \\
\hline Polycystic kidney disease & 1 \\
\hline Chromosomal anomalies & 4 \\
\hline Trisomy 13 & 2 \\
\hline Trisomy 18 & 2 \\
\hline Multiple congenital anomalies & 1 \\
\hline Haematology & 1 \\
\hline Acute myeloblastic leukaemia; trisomy 21 & 1 \\
\hline Limit of viability $<24$ weeks & 3 \\
\hline
\end{tabular}

PPHN, persistent pulmonary hypertension of the newborn; MAS, meconium aspiration syndrome.

written in accordance with the hospital and Ministry of Health policies, which state that the fact of DNR should be clearly recorded in the case notes, and humane care only should be given so long as the DNR order is in force. The DNR order could be revoked at any time by either the care giving team or by parents.
Once a DNR order was considered we classified patients into three groups:

- Group A: lesions incompatible with life

- Group B: lesions which will not allow meaningful survival

- Group C: end of treatment line; death inevitable.

In Groups $\mathrm{A}$ and $\mathrm{B}$, either no life support measures were initiated, or if initiated, could be withdrawn. Withdrawal of life support measures meant the discontinuation of ventilatory support, inotropes, and antibiotics. Anticonvulsants, sedation, fluids, feeds, and oxygen were not withdrawn.

In Group C, withdrawal of intensive care was the only possible option when counselling parents.

Data were gathered as to whether an antenatal diagnosis had been made and possible options discussed with one or both parents. The type of lesion was grouped according to system-cardiovascular disorders, renal anomalies, gastrointestinal, chromosomal, etc.

\section{RESULTS}

There were 659 admissions to the neonatal intensive care unit during this period, and 77 deaths $(12 \%)$. A DNR order was considered and written in $39(6 \%)$ infants. All were Muslim. In two of these the DNR order was subsequently revoked. Table 1 lists the major systems involved in these patients, with their diagnosis. Based on the diagnosis and perceived outcome, we classified the babies into three categories (see table 2).

Of these 39 patients, 28 died in our hospital, seven were sent back to the referring hospitals, and four were discharged home for terminal care. When patients were discharged home for terminal care, the fact was recorded on the discharge summary and child health card. All re-presented to the paediatric emergency department within one month of discharge in a terminal state, and died in hospital. Palliative care only was given. Of the seven sent back to referring hospitals, six died within one month and one is still alive at 6 months of age. In the two patients in whom the DNR order was initially written (polycystic kidney disease; severe chronic lung disease) but subsequently revoked, one died and one survived to discharge. He was lost to follow up.

The average time between birth and DNR was 10.6 (SD 14.8) days; DNR and death or discharge was 7.6 (SD 10.4)

\begin{tabular}{llllll|}
\hline Table 2 & Type of DNR & & \\
& & Outcome & \\
\cline { 3 - 5 } & & & Discharged \\
\cline { 3 - 5 } Type of DNR & No. & $\begin{array}{c}\text { Death at our } \\
\text { hospital }\end{array}$ & $\begin{array}{l}\text { Referring } \\
\text { hospital }\end{array}$ & Home \\
\hline Lesion incompatible with life & 15 & 14 & 1 & 0 \\
Lesion will not allow meaningful survival & 18 & 9 & 5 & 4 \\
End of treatment line: death inevitable & 6 & 5 & 1 & 0 \\
Total & 39 & 28 & 7 & 4 \\
\hline
\end{tabular}

Table 3 Time intervals

\begin{tabular}{lll}
\hline Type of DNR & Birth and DNR (days) & $\begin{array}{l}\text { DNR and death or discharge } \\
\text { (days) }\end{array}$ \\
\hline Lesion incompatible with life & $2(2.8)[1-11]$ & $5.7(9.1)[1-33]$ \\
Lesion will not allow meaningful survival & $12.3(14.8)[1-52]$ & $8.8(11.3)[1-44]$ \\
End of treatment line & $28.5(21.2)[6-56]$ & $7.7(12.8)[1-27]$ \\
\hline Values are mean (SD) [range]. & & \\
\hline
\end{tabular}


Table 4 Parental responses

\begin{tabular}{|c|c|c|c|c|}
\hline & No. & $\begin{array}{l}\text { Limitation/withdrawal } \\
\text { accepted }\end{array}$ & $\begin{array}{l}\text { Withdrawal } \\
\text { not accepted }\end{array}$ & $\begin{array}{l}\text { Not } \\
\text { applicable }\end{array}$ \\
\hline Ventilation initiated & 19 & 2 & 17 & 0 \\
\hline Ventilation not initiated & 20 & 14 & 0 & 6 \\
\hline
\end{tabular}

days. Based on the type of DNR, table 3 shows the time intervals. An average of 2.5 counselling sessions (range 1-5) was given to the parents before a consensus decision could be made regarding their infants.

Table 4 shows parental responses to withdrawal of support. In those in whom ventilation was initiated, only $11 \%$ of parents (2/19) allowed discontinuation of ventilatory support whereas $89 \%$ (17/19) refused or did not offer a definite opinion. In those in whom ventilation was not initiated, 70\% (14/ 20 ) agreed not to put their child on the ventilator and to discontinuation of inotropes and/or antibiotics if the child was receiving them at the time. In six of these 20 patients $(30 \%)$ the question of withdrawal did not arise (lethal trisomies, anencephaly, holoprosencephaly). Therefore none of these parents insisted on ventilation if deterioration occurred.

\section{DISCUSSION}

In Arab Muslim countries, the relationship between patient and physician is one of traditional paternalism, based on the principles of goodness and kindness. This is no different from Christianity, whose adherents are directed to do good to others in a spirit of love and kindness (Holy Bible, Matthew xxv, 35-45).

The Shariah law, which governs the citizens of Arab Muslim countries, is based on:

(1) The Quran-the Holy Text believed by Muslims to be the direct word of God.

(2) The Sunnah-the examples in word or deed of the Prophet Mohammed and incorporated into Islamic scriptures.

(3) The Ijtihad - the law of deductive logic. ${ }^{5}$ In this, learned scholars or Ulema are charged with interpreting and disseminating religious teachings. This may, of course, lead to differences in opinion between different scholars and therefore different interpretations of the law. To overcome this, many Ulema have concluded that in situations requiring specialist knowledge, for example, in medical practice, a new concept of "consensus edict" is preferable. In these rulings, the consensus groups have representations of Ulema and specialist clinicians, the latter providing the necessary background information. It is Ijtihad that provides the Muslim Sacred Law with its dynamism, allowing it to remain relevant while responding to new challenges and concerns that inevitably arise as a result of scientific advances. The issues at stake with regard to DNR are not clearly spelt out in the Quran and Sunnah, and have therefore to be interpreted from the Ijitihad (views and thinking of Muslim scholars and clergy).

One issue which we face in our patients is summarised as: "Whosoever takes a human life, for other than murder or corruption in the earth, it is as if he has taken the life of all mankind" ${ }^{6}$ No one is therefore authorised to deliberately end life, either one's own or that of another human being. However, reducing suffering by analgesia, even if death is thereby hastened, is permitted, based on the central teaching that "actions are to be judged by their intentions". Withholding nutrition and fluid therapy is not allowed. We therefore continued fluids and feeds in all our patients.
In 1987 a US based Muslim thinker expressed the view that unnecessary artificial prolongation of life is not in keeping with the spirit of Islam, unless there is evidence that a reasonable quality of life will result. ${ }^{7}$ This led us to the question of whether euthanasia would be permissible or acceptable. Active euthanasia is always illegal, and passive euthanasia also is not generally accepted despite certain religious writers' views on the issue and a professional code of practice recommending that physicians should not impose futile care in an attitude of unreasonable persistence. However, others are of the view that no situation is considered hopeless for Muslim believers who believe in the omnipotence of an Almighty God. Withholding or withdrawing treatment from any patient is therefore never easy and cannot be generalised. Cultural, social, and religious issues have to be taken into account when making decisions. We remind patients of God's omnipotence, and the transient nature of our time on earth and the abiding reality of spending eternity with God. Whatever we may do, the ultimate fact is that God determines the time of death, and we are only God's instruments for the good of the patient.

We very rarely portray a wholly negative attitude, but show guarded optimism tempered with scientific reality, where we discuss the real possibility of worse to come-either slowly or catastrophically. When DNR decisions are being discussed with the parents, we nearly always ask them if they would like other close family members to be present. The presence of an elderly male, who may be a close relative or a cleric, often brings relief to parents bearing the burden of having to cope with a dying child and make decisions at the same time. More often, through this dynamic dialogue, we have to gauge parents' and families' wishes, even though this is virtually never articulated. When the question of withdrawal of life support measures is raised (turning off the ventilator), we meet with near universal refusal (89\%). Parents and extended family do not want to be seen as having acquiesced in their child's demise. In contrast, when the child is not ventilated but a decision of DNR or limiting vital support measures is made, none have objections to limiting therapy. We always say that "In our opinion, and if this was my child, I would not put the child on the ventilator". Parents often acquiesce to this either silently, or by saying "You are the doctor and nurse. You do what is best for my child". We interpret this as parents not wanting to make a life or death decision themselves, but are happy to accept transferring the responsibility onto a person in authority. This is where we have found extended family support and the presence of clergy extremely helpful. Asking parents alone to make this decision is not appropriate or acceptable. Even asking them to be part of the decision making process, when withdrawal of therapy is medically warranted, may be inappropriate in this culture.

This approach may not be very different from the practice in certain European countries. In a French study, in which a large number of intensive care units were polled, it was shown that withholding or withdrawing life support is widely practiced, despite prohibition by legislation. ${ }^{8}$ In a study of various neonatal units in Europe about the policy of DNR and ethical decision making, it was found that in Great Britain, parents are explicitly involved in this process by open participation (78\% units surveyed) or assume full responsibility for their choice $(11 \%)$. In contrast, none of the French units reported 
open involvement of parents in decision making, although parental views are always "indirectly sounded out and taken into account". In Sweden, $68 \%$ of units follow this policy of indirectly sounding out parents' wishes, and in $26 \%$ they are involved in the decision making process. ${ }^{9}$

Contemporary practice and moral law in the western world is therefore not very different. Ethical decisions primarily reflect the interest of the infant. Interests of parents, other family members, or society at large are secondary. ${ }^{10}$ This leads to differences in opinion as to what constitutes the best interest of the child and who is to administer this right. Parents are expected to act on behalf of their child and make decisions for them. In 1981 Dr Leonard Arthur was tried for attempted murder, for acting in the belief that non-treatment of an infant with trisomy 21 who had been abandoned by his parents was justified. ${ }^{11}$ Shared responsibility between neonatologists, obstetricians, and parents is probably the best way forward in a society with a high level of education and concept of individual rights. This may not be so in other societies, and trust in and integrity of the care givers would therefore play a vital role in the decision making process. Attempts by lawmakers or administration to force treatment, which may be considered by parents and physicians to be futile or inhumane, may lead to an atmosphere of distrust and fear, as happened in the USA in the 1980s with the Baby Doe regulations. $^{12}$

The main findings in our study were that 36\% (28/77) of the deaths in our NICU were preceded by a DNR order. This is lower than the $67.9 \%$ reported by Torres and Garcia, ${ }^{13}$ the $50-55 \%$ in a population of surgical neonates from the Netherlands and the USA, ${ }^{14}$ and the $46.2 \%$ in a Nigerian study of perinatal deaths. ${ }^{15}$ This may be related to the fact that parents are reluctant to consider or acquiesce to a DNR order, as they feel that in matters of life and death only God decides and we are His chosen instruments. All should therefore be done to support the infant and the rest left to God.

Only two of $19(11 \%)$ parents of patients in whom ventilation was ongoing permitted withdrawal. The rest silently acquiescenced in the fact that withdrawal was necessary, but it was never explicitly articulated. We consider this as "indirectly sounding out their views and taking them into account" as discussed earlier. They were aware that some form of intensive care was being withdrawn and we were keeping their child as comfortable and pain free as possible. Ventilation, of course, was not completely withdrawn, though in seven of the 17 patients, ventilation variables were turned down-that is, supplemental oxygen was gradually withdrawn and inflation pressure was reduced. Parents were aware of this by seeing the "numbers" on the display panels. Is this the right way of going about withdrawal of therapy in ventilated patients, especially considering that the local relationship between patient and physician is one of traditional paternalism? We do not know and this is a constantly evolving issue. However, we strongly feel that we are acting in the best interest of parents, considering all scientific knowledge and the resources available in the community.

Most of the DNR decisions in the unit involved malformations $(24 / 39,62 \%)$. Withholding therapy was accepted better in these patients, especially if an antenatal diagnosis had been made. The general feeling of the parents was one of relief once the decision was made not to resuscitate or their child died.

We do not resuscitate infants with gestational age 24 weeks or less, and we consider a limit of viability as 25 weeks and above. This is based on a realistic assessment of the resources available locally, and from both short term and long term data reported from western countries and our own follow up clinic. This differs from units in the USA where, in a survey of 3056 neonatologists (1131 complete responses), $62 \%$ would give full intensive care (ventilate and administer surfactant) to infants at 23 weeks and $78 \%$ to infants at 24 weeks. ${ }^{16}$
From the viewpoint of the care giving staff, we noticed that neonatal experience accentuated the disinclination to resuscitate a "high risk baby". Year 1 and 2 residents were more likely to offer no opinion or want to resuscitate these infants compared to year 3 residents or "fellows" on the NICU. Religious preferences were not an issue as all residents are Muslim. Factors in the junior residents' views on resuscitatation were the "role of physicians to save lives", the "inability to prognosticate the outcome" which was not based on scientific literature but based on the omnipotence of God, and that we should not play at being God. We did not track their attitudes to DNR through their levels of training. "Senior nurses" ( those who have worked for more than two years on the NICU) held strong views on the issue and nearly always agreed with the DNR decisions. In fact in more than $50 \%$ of group B and C patients, the DNR decision was first broached with the neonatologist by the bedside nurse.

We conclude that it is necessary to take decisions on vital support limitation or withdrawal of therapy in neonates, especially in malformed infants, or when they are critically ill. To accomplish this, we evaluated individual patients' prognoses, the acceptable "grassroot" standards of ethical care in the community, and the child's and family's best interest. It is not acceptable, in this community, to ask parents to make decisions regarding life support of their infants; it is preferable to make the decision for them, based on published scientific evidence in the context of resources and support services locally available and gauging their response in discussions with them.

\section{Authors' affiliations}

D E da Costa, H Ghazal, Saleh Al Khusaiby, Department of Child Health, Royal Hospital, Ministry of Health, Sultanate of Oman

\section{REFERENCES}

1 Taylor HG, Klein N, Hack M. School-age consequences of birth weight less than $750 \mathrm{~g}$ : a review and update. Dev Neuropsychol 2000; 17:289-321

2 Hack M, Fanaroff AA. Outcomes of children of extremely low birth weight and gestational age in the 1990s. Semin Neonatol 2000;5:89-106.

3 Doyal L, Wilsher D. Towards guidelines for withholding and withdrawa of life prolonging treatment in neonatal medicine. Arch Dis Child Fetal Neonatal Ed 1994:70:F66-F70.

4 Lantos JD, Tyson JE, Allen A, et al. Withholding and withdrawing life sustaining treatment in neonatal intensive care: issues for the 1990s. Arch Dis Child Fetal Neonatal Ed 1994;71:F218-F223.

5 Gatrad AR, Sheik A. Medical ethics and Islam: principles and practice. Arch Dis Child 2001;84:72-5.

6 Ali YA. The meaning of the glorious Quran. Dar Al Kitab 1938;5:32.

7 Rehman F. Health and medicine in the Islamic tradition. Chicago: ABC, 1998;108-9.

8 Ferrand E, Robert R, Ingrand P, Lemaire F. Witholding and withdrawal of life support in intensive care units in France: a prospective study. Lancet $2001 ; 357: 9-14$

9 Cuttini $M$, Rebagliato $M$, Bortoli $P$, et al. Parental visiting communication and participation in ethical decisions: a comparison of neonatal unit policies in Europe. Arch Dis Child Fetal Neonatal Ed 1999;81:F84-F91.

10 Jonsen A. Critical issues in newborn intensive care: a conference report and policy proposal. Pediatrics 1975;55:756-85.

11 Anonymous. Dr Leonard Arthur: his trial and its implications [editorial]. BM 1981;283:1340

12 Lantos JD. Baby Doe five years later: implications for child health. $N$ Engl J Med 1987:317:444-7.

13 Tejedor Torres JC, Garcia AL. Making ethical decisions of limiting vital support to critical newborns. Ann Esp Pediatr 1997;46:53-9.

14 Caniano DA, Hazebroek FW, DenBesten KE, Tibboel D. End of life decisions for surgical neonates: experiences in The Netherlands and United States. J Pediatr Surg 1995;30:1420-4.

15 Airede KI. Should we resuscitate: ethical dilemmas. Ann Trop Paediatr 1991;11:169-74.

16 Sanders MR, Donohue PK, Oberdorf MA, et al. Impact of the perception of viability on resource allocation in the neonatal intensive care unit. $J$ Perinatol 1998;18:347-51. 


\section{COMMENTARY}

Muslim parents in the United Kingdom are much more knowledgeable about their rights and want to get involved with decision making more and more. The support of the extended family, although still strong, is slowly breaking down, and many more can now communicate in English. Although neonatal mortality in the United Kingdom is different from that in Oman, difficulties can arise, particularly when carers of a different faith are perceived not to understand the Islamic viewpoint. There is in the Muslim "psyche" the notion that non-Muslim professionals do not value a Muslim's life as much as another. However, more and more Muslim mothers are agreeing to antenatal screening and terminations, particularly if they have already had a child with a congenital problem.

In my experience, parents are very much involved, with some support still being provided by the extended family. The difficult decision of DNR is less likely to be accepted by Muslim parents and relatives than the host population in the United Kingdom. In this decision making process, nursing staff play a vital role; in the United Kingdom nursing is still considered by Muslims to be a somewhat "liberal" profession not conforming to Islamic ways. We therefore do not have the same religious support as in Oman; 50\% of the nursing staff in this study was Muslim. One of the ways around the difficult problem of communicating a decision not to resuscitate is to make it clear to parents that the professionals do not want them to make the final decision to end life. Rather that the parents allow the carers to make a judgment and, if necessary, decide to withdraw life support. This can be clearly inserted in the case notes of the neonate. For this strategy to work, the full trust of the family unit has to be gained before the subject can be approached.

The local population in developing countries hold doctors in high esteem, particularly if trained in the West. Unfortunately in the United Kingdom, generally, doctors are not respected to the same degree and so the question of trust becomes all the more important. Silent acquiescence by the parents in the withdrawal of treatment, as happens in Oman, would be fraught with medicolegal problems here if not explicitly articulated and agreed to by the parents.

From time to time it can be useful to have a second opinion from a practising Muslim paediatrician, as has happened in the West Midlands. The simple greeting of "Assalamualaikum" often breaks down the immediate barrier and facilitates further discussion. The literature on health and Islamic issues continues to proliferate slowly, as some professionals take an interest and begin to understand other religions and cultures.

This paper raises the important issue of training young doctors in ethical issues. This, I believe, should start in medical school, as has been suggested by the General Medical Council in its document "Tomorrow's doctors". Such training should include ethical issues of other cultures.

A R Gatrad
Walsall Hospitals NHS Trust, Manor Hospital, Moat Road, West Midlands WS2 9PS, UK 